\title{
UNIFORM-ULTIMATE POISSON BOUNDEDNESS OF SOLUTIONS OF P-PERTURBED SYSTEMS OF DIFFERENTIAL EQUATIONS
}

\author{
KIRILL S. LAPIN AND CEMIL TUNÇ
}

Received 07 April, 2020

\begin{abstract}
The concept of $P$-perturbed systems with respect to given system of ordinary differential equations is introduced. Certain sufficient conditions for uniform-ultimate Poisson boundedness of solutions of $P$-perturbed systems are obtained by using the method of Lyapunov functions. In conclusion, an interesting application of the above sufficient conditions to the standard closed electric RLC circuit with nonlinear elements is given.
\end{abstract}

2010 Mathematics Subject Classification: 34C11; 34D20

Keywords: P-perturbed systems, differential equations, uniform-ultimate Poisson boundedness, Lyapunov function

\section{INTRODUCTION AND PRELIMINARIES}

The theory of boundedness of solutions in the Poisson sense was developed in [2-5]. It generalizes the usual theory of boundedness of solutions for systems of differential equations [7]. The concept of Poisson boundedness of solutions is characterized by the fact that the solution may not be completely contained in some ball in the phase space, but has the property of a countable number of times of recurrence into this ball.

The paper is devoted to finding sufficient conditions for uniform-ultimate Poisson boundedness of solutions of $P$-perturbed systems. By a $P$-perturbed system we mean a system whose right-hand side is equal to the right-hand side of a given system multiplied by the function $1 /(g(t)+\alpha(t))$, where $g(t)$ is any continuously differentiable periodic function, for which $\min g(t)=0$ and $\max g(t)>0$, and $\alpha(t)$ is any positive decreasing differentiable function satisfying the condition $\alpha(t) \rightarrow 0$ for $t \rightarrow+\infty$. The need to research $P$-perturbed systems with respect to given systems occurs when, under the influence of external forces, the modulus of the vector of the phase velocity of a given system begins to perform forced oscillations, the amplitude of which increases with time indefinitely.

The paper begins with consideration of the necessary information related to the concept of Poisson uniform-ultimate boundedness of solutions. Next, we introduce the concept of a $P$-perturbed system with respect to a given system. A sufficient 
condition is obtained for uniform-ultimate Poisson boundedness of solutions of a $P$ perturbed system with respect to any linear system with constant coefficients. Then, using this sufficient condition and the method of Lyapunov functions, we obtain a sufficient condition for the uniform-ultimate Poisson boundedness of solutions of a $P$ perturbed system with respect to an arbitrary system. In the conclusion of this paper we give an interesting application of the above sufficient conditions to the standard closed electric RLC circuit with nonlinear elements. Now let us pass to the exact definitions and formulations.

We consider a system of differential equations in $n \geqslant 1$ variables

$$
\frac{d x}{d t}=F(t, x), \quad F(t, x)=\left(F_{1}(t, x), \ldots, F_{n}(t, x)\right)^{T},
$$

where $F: \mathbb{R}^{+} \times \mathbb{R}^{n} \rightarrow \mathbb{R}^{n}$ is any continuous vector function. Here $\mathbb{R}^{+}$denotes the set $\{t \in \mathbb{R} \mid t \geqslant 0\}$. It is assumed that all solutions of system (1.1) are extendable to the whole semi-axis $\mathbb{R}^{+}$. The uniqueness of the solution of the Cauchy problem for system (1.1) is not required.

Further, under $\|\cdot\|$ everywhere, we mean the usual Euclidean norm for vectors and matrices. The solution $x=x(t)$ of the system (1.1) passing through the point $\left(t_{0}, x_{0}\right) \in \mathbb{R}^{+} \times \mathbb{R}^{n}$ will be written as $x=x\left(t, t_{0}, x_{0}\right)$. For any $t_{0} \in \mathbb{R}^{+}$, we will further denote by $\mathbb{R}^{+}\left(t_{0}\right)$ the set $\left\{t \in \mathbb{R} \mid t \geqslant t_{0}\right\}$. Any non-negative increasing sequence $\tau=\left(\tau_{i}\right)_{i \geqslant 1}$ of real numbers for which the condition $\lim _{i \rightarrow+\infty} \tau_{i}=+\infty$ is satisfied will be called a $P$-sequence. For each $P$-sequence $\tau=\left(\tau_{i}\right)_{i \geqslant 1}$, we will further denote by $M(\tau)$ the set $\bigcup_{i=1}^{\infty}\left[\tau_{2 i-1}, \tau_{2 i}\right]$.

Recall [3] that the solutions of the system (1.1) are said to be uniform-ultimately Poisson bounded if there exist a number $B>0$ and a $P$-sequence $\tau=\left(\tau_{i}\right)_{i \geqslant 1}$, such that for any $\alpha \geqslant 0$ there exist number $T \geqslant 0$ such that for any solution $x=x\left(t, t_{0}, x_{0}\right)$ of system (1.1), where $t_{0} \in M(\tau)$ and $\left\|x_{0}\right\| \leqslant \alpha$, the condition $\left\|x\left(t, t_{0}, x_{0}\right)\right\|<B$ is satisfied for all $t \in \mathbb{R}^{+}\left(t_{0}+T\right) \cap M(\tau)$. In the case where it is required to specify the corresponding $P$-sequence $\tau=\left(\tau_{i}\right)_{i \geqslant 1}$, it says that the solutions of the system (1.1) are uniform-ultimately Poisson bounded with respect to the $P$-sequence $\tau=\left(\tau_{i}\right)_{i \geqslant 1}$.

For comparison, it is also useful to recall [7] that the solutions of the system (1.1) are said to be uniform-ultimately bounded if for the system (1.1) there is a number $B>0$, and for any $\alpha \geqslant 0$ there exists a number $T \geqslant 0$ such that for any solution $x=$ $x\left(t, t_{0}, x_{0}\right)$ of system $(1.1)$, where $t_{0} \in \mathbb{R}^{+}$and $\left\|x_{0}\right\| \leqslant \alpha$, the condition $\left\|x\left(t, t_{0}, x_{0}\right)\right\|<$ $B$ is satisfied for all $t \in \mathbb{R}^{+}\left(t_{0}+T\right)$.

It can be seen that if the solutions of the system (1.1) are uniform-ultimately bounded, then they are uniform-ultimately Poisson bounded.

In [5], based on the Lyapunov vector function method [6], the following sufficient condition for the uniform-ultimate Poisson boundedness of solutions of the system (1.1) was obtained. 
Theorem 1. Suppose that for system (1.1) there exist a P-sequence $\tau=\left(\tau_{i}\right)_{i \geqslant 1}$, continuously differentiable function $V: \mathbb{R}^{+} \times \mathbb{R}^{n} \rightarrow \mathbb{R}^{+}$, an increasing function $a$ : $\mathbb{R}^{+} \rightarrow \mathbb{R}^{+}$, a non-decreasing function $b: \mathbb{R}^{+} \rightarrow \mathbb{R}^{+}$, which has the property $b(r) \rightarrow$ $+\infty$ for $r \rightarrow+\infty$, and continuous function $f: \mathbb{R}^{+} \times \mathbb{R} \rightarrow \mathbb{R}$ that satisfy the following conditions:

(1) $b(\|x\|) \leqslant V(t, x) \leqslant a(\|x\|)$ for all $(t, x) \in M(\tau) \times \mathbb{R}^{n}$.

(2) $\dot{V}(t, x) \leqslant f(t, V(t, x))$ for all $(t, x) \in \mathbb{R}^{+} \times \mathbb{R}^{n}$.

(3) The solutions of the equation $\dot{\xi}=f(t, \xi)$, called the comparison equation for the system (1.1), are uniform-ultimately Poisson bounded with respect to the $P$-sequence $\tau=\left(\tau_{i}\right)_{i \geqslant 1}$.

Then the solutions of the system (1.1) are uniform-ultimately Poisson bounded.

\section{RESULTS}

We now introduce the concept of a $P$-perturbed system with respect to the system (1.1). Let $g(t)$ be any continuously differentiable periodic function defined on $\mathbb{R}^{+}$satisfying the conditions $\min g(t)=0$ and $\max g(t)>0$. From properties of the function $g(t)$, it follows that $g^{\prime}(t)$ is a continuous periodic function for which the conditions $\max g^{\prime}(t)>0$ and $\min g^{\prime}(t)<0$ are satisfied. Moreover, let $\alpha(t)>0$ be any differentiable decreasing function defined on $\mathbb{R}^{+}$for which the condition $\alpha(t) \rightarrow 0$ is fulfilled as $t \rightarrow+\infty$. Simple examples of such functions are $g(t)=1+\sin (t)$ and $\alpha(t)=e^{-t}$.

We will call a system that has the form

$$
\frac{d x}{d t}=\frac{1}{g(t)+\alpha(t)} F(t, x),
$$

where $g(t)$ and $\alpha(t)$ are any of the above functions, a $P$-perturbed system with respect to the system (1.1) or, if misunderstandings are excluded, just a $P$-perturbed system.

Consider a $P$-perturbed system with respect to an arbitrary linear system $\dot{x}=A x$, $x \in \mathbb{R}^{n}, n \geqslant 1$, i.e., consider the system

$$
\frac{d x}{d t}=\frac{1}{g(t)+\alpha(t)} A x,
$$

where $A$ is a constant real $(n \times n)$ - matrix.

Theorem 2. If the eigenvalues $\lambda_{1}, \ldots, \lambda_{n}$ of the matrix $A$ on the right-hand side of the P-perturbed system (2.2) satisfy the condition $\operatorname{Re}\left(\lambda_{i}\right)<-\max g^{\prime}(t)$ for all $1 \leqslant i \leqslant n$, then solutions of this P-perturbed system are uniform-ultimately Poisson bounded.

Proof. First we define the Lyapunov function $V(t, x)$. To do this, we will make the change of variables $x=S y$, where $y=\left(y_{1}, \ldots, y_{n}\right)^{T} \in \mathbb{C}^{n}, S$ is a complex $(n \times n)$ matrix for which $S^{-1} A S=B$ is an upper triangular complex matrix, on the main diagonal of which are the eigenvalues $\lambda_{1}, \ldots, \lambda_{n}$ of matrix $A$. Note that the above 
change of variables $x=S y$ is equivalent to changing the variables $x=\bar{S} \bar{y}$, where $\bar{y}$ is a complex number conjugate to $y$ and $\bar{S}=\left(\bar{s}_{i j}\right)$ is the matrix conjugate to $S=\left(s_{i j}\right)$. It is clear that for the above replacement of variables $x=\bar{S} \bar{y}$, the equality $(\bar{S})^{-1} A \bar{S}=\bar{B}$ is true, where $\bar{B}=\left(\bar{b}_{i j}\right)$ is an upper triangular matrix, conjugate to the matrix $B=\left(b_{i j}\right)$. It is known (see, for example, [1]), that for any real number $b>0$ there exists a change of variables $x=S y$ such that for all $1 \leqslant i<j \leqslant n$ the inequality $\left|b_{i j}\right|<b$ is valid, where $\left|b_{i j}\right|$ is the modulus of the complex number $b_{i j}$. Define the function $V: \mathbb{R}^{+} \times \mathbb{R}^{n} \rightarrow \mathbb{R}$ by

$$
\begin{aligned}
V(t, x)= & (g(t)+\alpha(t)) \cdot\left(S^{-1} x,(\bar{S})^{-1} x\right)=(g(t)+\alpha(t)) \cdot(y, \bar{y}) \\
& =(g(t)+\alpha(t)) \sum_{i=1}^{n} y_{i} \bar{y}_{i}=(g(t)+\alpha(t))\|y\|^{2}=(g(t)+\alpha(t)) \sum_{i=1}^{n}\left|y_{i}\right|^{2} \geqslant 0,
\end{aligned}
$$

where $($,$) is a scalar multiplication in \mathbb{C}^{n}$. Consider, for any fixed $0<\gamma<\max g(t)$, an increasing sequence $\vartheta_{1}<\vartheta_{2}<\cdots<\vartheta_{i}<\ldots$ of all roots of the equation $g(t)+$ $\alpha(t)=\gamma$. Let $\vartheta_{k}$ be the first root for which on the segment $\left[\vartheta_{k}, \vartheta_{k+1}\right]$ the condition $g(t)+\alpha(t) \geqslant \gamma$ is satisfied. Define the increasing sequence $\tau_{1}<\tau_{2}<\cdots<\tau_{i}<\ldots$, assuming $\tau_{i}=\vartheta_{i+k-1}, i \geqslant 1$. From the properties of the functions $g(t)$ and $\alpha(t)$, it follows that $\lim _{i \rightarrow+\infty} \tau_{i}=+\infty$ and, therefore, $\tau=\left(\tau_{i}\right)_{i \geqslant 1}$ is a $P$-sequence. It is easy to check that for any $t \in \mathbb{R}^{+}\left(\tau_{1}\right)$ and $x \in \mathbb{R}^{n}$, the inequality $V(t, x) \leqslant a(\|x\|)$ holds, where $a(r)=(\max g(t)+\max \alpha(t))\left(\left\|S^{-1}\right\| r\right)^{2}$. Since on each closed interval $\left[\tau_{2 i-1}, \tau_{2 i}\right]$, $i \geqslant 1, \gamma \leqslant g(t)+\alpha(t)$, then for all $(t, x) \in M(\tau) \times \mathbb{R}^{n}$ we get $b(\|x\|) \leqslant V(t, x)$, where $b(r)=\gamma \cdot(r /\|S\|)^{2}$. It follows that the double inequality $b(\|x\|) \leqslant V(t, x) \leqslant a(\|x\|)$ holds for all $(t, x) \in M(\tau) \times \mathbb{R}^{n}$. For the derivative $\dot{V}(t, x)$ of the function $V(t, x)$ along solutions of the of the system (2.2), we have the following equalities:

$$
\begin{aligned}
\dot{V}(t, x)= & \left(g^{\prime}(t)+\alpha^{\prime}(t)\right)\|y\|^{2}+\left(S^{-1} A x,(\bar{S})^{-1} x\right)+\left(S^{-1} x,(\bar{S})^{-1} A x\right) \\
= & \left(g^{\prime}(t)+\alpha^{\prime}(t)\right)\|y\|^{2}+\left(S^{-1} A S y,(\bar{S})^{-1} \bar{S} \bar{y}\right)+\left(S^{-1} S y,(\bar{S})^{-1} A \bar{S} \bar{y}\right) \\
= & \left(g^{\prime}(t)+\alpha^{\prime}(t)\right)\|y\|^{2}+(B y, \bar{y})+(y, \bar{B} \bar{y})=\left(g^{\prime}(t)+\alpha^{\prime}(t)\right)\|y\|^{2} \\
+ & \left(\sum_{i=1}^{n} \lambda_{i} y_{i} \bar{y}_{i}+\sum_{1 \leqslant i<j \leqslant n} b_{i j} y_{j} \bar{y}_{i}\right)+\left(\sum_{i=1}^{n} \bar{\lambda}_{i} y_{i} \bar{y}_{i}+\sum_{1 \leqslant i<j \leqslant n} \bar{b}_{i j} \bar{y}_{j} y_{i}\right) \\
& =\left(g^{\prime}(t)+\alpha^{\prime}(t)\right)\|y\|^{2}+\sum_{i=1}^{n}\left(\lambda_{i}+\bar{\lambda}_{i}\right) y_{i} \bar{y}_{i}+\sum_{1 \leqslant i<j \leqslant n}\left(b_{i j} y_{j} \bar{y}_{i}+\bar{b}_{i j} \bar{y}_{j} y_{i}\right) .
\end{aligned}
$$

Since $\alpha^{\prime}(t)<0$ for all $t \geqslant 0$ and $\max g^{\prime}(t)>0$, we have $g^{\prime}(t)+\alpha^{\prime}(t)<\max g^{\prime}(t)$ for all $t \geqslant 0$. Under the conditions of the theorem, $\operatorname{Re}\left(\lambda_{i}\right)<-\max g^{\prime}(t)$ for any $1 \leqslant i \leqslant n$. Therefore, we have $\lambda_{i}+\bar{\lambda}_{i}=2 \operatorname{Re}\left(\lambda_{i}\right)<-2 \max g^{\prime}(t)$. Because $\bar{b}_{i j} y_{j} \bar{y}_{i}=\bar{b}_{i j} \bar{y}_{j} y_{i}$, we get

$$
\sum_{1 \leqslant i<j \leqslant n}\left(b_{i j} y_{j} \bar{y}_{i}+\bar{b}_{i j} \bar{y}_{j} y_{i}\right)=\sum_{1 \leqslant i<j \leqslant n} 2 \operatorname{Re}\left(b_{i j} y_{j} \bar{y}_{i}\right) \leqslant \sum_{1 \leqslant i<j \leqslant n} 2\left|b_{i j} y_{j} \bar{y}_{i}\right|
$$




$$
\begin{array}{r}
=\sum_{1 \leqslant i<j \leqslant n} 2\left|b_{i j}\right|\left|y_{j}\right|\left|\bar{y}_{i}\right| \leqslant \sum_{1 \leqslant i<j \leqslant n}\left|b_{i j}\right|\left(\left|y_{j}\right|^{2}+\left|y_{i}\right|^{2}\right) \leqslant \sum_{1 \leqslant i<j \leqslant n}\left|b_{i j}\right|\|y\|^{2} \leqslant \\
\leqslant \sum_{1 \leqslant i<j \leqslant n} \mu\|y\|^{2}=\frac{1}{2} n(n-1) \mu\|y\|^{2},
\end{array}
$$

where $\mu>0$ is a preassigned number for which $\left|b_{i j}\right|<\mu$. From the above inequalities we get $\dot{V}(t, x) \leqslant\left(-\max g^{\prime}(t)+(1 / 2) n(n-1) \mu\right)\|y\|^{2}$. If we choose the number $\mu>$ 0 so that $\mu<\left(2 \max g^{\prime}(t)\right) /(n(n-1))$, then we get $\dot{V}(t, x) \leqslant-\psi\|y\|^{2}$, where $\psi=$ $\max g^{\prime}(t)-(1 / 2) n(n-1) \mu>0$. From the inequality $\dot{V}(t, x) \leqslant-\psi\|y\|^{2}$, it follows that

$$
\dot{V}(t, x) \leqslant-\frac{\psi}{g(t)+\alpha(t)} V(t, x) .
$$

Because $-\psi /(g(t)+\alpha(t)) \leqslant-\psi / \max (g(t)+\alpha(t))$, we obtain the inequality

$$
\dot{V}(t, x) \leqslant-c V(t, x),
$$

where $c=\psi / \max (g(t)+\alpha(t))>0$. This inequality suggests that the comparison equation for the system (2.2) is $\dot{\xi}=-c \xi$. We can establish by the Lyapunov functions method that the solutions of equation $\dot{\xi}=-c \xi$ are uniform-ultimately bounded. For example, we can take the function $W(\xi)=\xi^{2}$ as a Lyapunov function for the equation $\dot{\xi}=-c \xi$. Since the solutions of the equation $\dot{\xi}=-c \xi$ are uniform-ultimately bounded, then they are uniform-ultimately Poisson bounded with respect to the above $P$-sequence $\tau=\left(\tau_{i}\right)_{i \geqslant 1}$. Thus, for the $P$-perturbed system (2.2), all conditions of Theorem 1 are fulfilled and, therefore, the solutions of this $P$-perturbed system are uniform-ultimately bounded.

Obviously, if in the conditions of Theorem 1 we take $n=1$, then we obtain the following statement.

Corollary 1. Suppose that for P-perturbed equation

$$
\frac{d x}{d t}=\frac{1}{g(t)+\alpha(t)} a x
$$

with respect to linear equation $\dot{x}=a x$, where $a \in \mathbb{R}$ satisfies condition $a<-\max g^{\prime}(t)$. Then solutions of this P-perturbed equation is uniform-ultimately Poisson bounded.

We obtain, using the method of Lyapunov functions and Corollary 1, the sufficient condition for uniform-ultimate Poisson boundedness of solutions of $P$-perturbed system (2.1).

Theorem 3. Suppose that for the P-perturbed system (2.1) there exists a differentiable function $V: \mathbb{R}^{+} \times \mathbb{R}^{n} \rightarrow \mathbb{R}$, constants $c_{1}>0, c_{2}>0, c_{3}>0$, a continuously differentiable periodic function $g: \mathbb{R}^{+} \rightarrow \mathbb{R}^{+}$, satisfying the requirements $\min g(t)=0$, $\max g(t)>0$, and a differentiable decreasing function $\alpha: \mathbb{R}^{+} \rightarrow \mathbb{R}^{+}, \alpha(t)>0, t \in \mathbb{R}^{+}$ satisfying $\alpha(t) \rightarrow 0$ with $t \rightarrow+\infty$. For all $t \in \mathbb{R}^{+}$and $x \in \mathbb{R}^{n}$ the following conditions are assumed: 
(1) $c_{1}\|x\|^{2} \leqslant V(t, x) \leqslant c_{2}\|x\|^{2}$

(2) $(g(t)+\alpha(t)) \frac{\partial V(t, x)}{\partial t}+\left(\frac{\partial V(t, x)}{\partial x}, F(t, x)\right) \leqslant-c_{3}\|x\|^{2}$;

(3) $\max g^{\prime}(t)<\frac{c_{3}}{2 c_{2}}$.

Then solutions of the P-perturbed system (2.1) are uniform-ultimately Poisson bounded.

Proof. We define the function $W: \mathbb{R}^{+} \times \mathbb{R}^{n} \rightarrow \mathbb{R}$ by

$$
W(t, x)=(g(t)+\alpha(t)) V(t, x)
$$

. For any fixed $0<\gamma<\max g(t)$ we consider an increasing sequence $\vartheta_{1}<\vartheta_{2}<\cdots<$ $\vartheta_{i}<\ldots$ of all roots of the equation $g(t)+\alpha(t)=\gamma$. Let $\vartheta_{k}$ be the first of the above roots, for which on the segment $\left[\vartheta_{k}, \vartheta_{k+1}\right]$ the condition $g(t)+\alpha(t) \geqslant \gamma$ is satisfied. We define an increasing sequence $\tau_{1}<\tau_{2}<\cdots<\tau_{i}<\ldots$ by setting $\tau_{i}=\vartheta_{i+k-1}$, $i \geqslant 1$. From the properties of functions $g(t)$ and $\alpha(t)$, it follows that $\lim _{i \rightarrow+\infty} \tau_{i}=+\infty$ and, therefore, $\tau=\left(\tau_{i}\right)_{i \geqslant 1}$ is a $P$-sequence. Condition (1) of the theorem follows that the inequality $W(x, t) \leqslant a(\|x\|)$ holds for any $t \in\left[\tau_{1}, \infty\right]$ and $x \in \mathbb{R}^{n}$, where $a(r)=$ $\max (g(t) \alpha(t)) c_{2} r^{2}$. It is easy to see that on each closed interval $\left[\tau_{2 i-1}, \tau_{2 i}\right], i \geqslant 1$, the inequality $\gamma \leqslant g(t) \alpha(t)$ holds. Therefore, by using condition (1) of the theorem, we obtain that the inequality $b(\|x\|) \leqslant W(t, x)$ is true for all $(t, x) \in M(\tau) \times \mathbb{R}^{n}$, where $b(r)=\gamma c_{1} r^{2}$, it follows that the double inequality

$$
b(\|x\|) \leqslant W(t, x) \leqslant a\|x\|)
$$

holds for all $(t, x) \in M(\tau) \times \mathbb{R}^{n}$. The derivative $\dot{W}(t, x)$ of $W(t, x)$ along solutions of the system (2.1) becomes

$$
\begin{aligned}
\dot{W}(t, x) & =\frac{\partial W(t, x)}{\partial t}+\left(\frac{\partial W(t, x)}{\partial x}, \frac{F(t, x)}{g(t)+\alpha(t)}\right) \\
& =\left(g^{\prime}(t)+\alpha^{\prime}(t)\right) V(t, x)+(g(t)+\alpha(t)) \frac{\partial V(t, x)}{\partial t}+\left(\frac{\partial V(t, x)}{\partial x}, F(t, x)\right) .
\end{aligned}
$$

Using conditions (1) and (2) of the theorem, and also considering that $\alpha^{\prime}(t)<0$, we obtain the inequality

$$
\left.\dot{W}(t, x) \leqslant\left(\max g^{\prime}(t)\right) V(t, x)-c_{3}\|x\|^{2} \leqslant \frac{1}{g(t)+\alpha(t)}\left(\max g^{\prime}(t)\right)-\frac{c_{3}}{c_{2}}\right) W(t, x) .
$$

This inequality means that the equation

$$
\left.\dot{\xi}=\frac{1}{g(t)+\alpha(t)}\left(\max g^{\prime}(t)\right)-\frac{c_{3}}{c_{2}}\right) \xi
$$

is the comparison equation for system (2.1). Since $\left.\left.\max g^{\prime}(t)\right)-\left(c_{3} / c_{2}\right)<-\max g^{\prime}(t)\right)$ holds by condition (3) of the theorem, using Corollary 1 we find that solutions of the 
comparison equation (2.3) are uniform-ultimately Poisson bounded. Thus, all conditions of Theorem 1 are fulfilled and, therefore, solutions of $P$-perturbed system (2.1) are uniform-ultimately Poisson bounded.

Let us consider an interesting application of Theorem 3 to the standard closed electric $R L C$ circuit with nonlinear elements. The equation of the charge $x$ (with $x^{\prime}$ being the current) is the following nonlinear ordinary differential equation of second order:

$$
L x^{\prime \prime}+R x^{\prime}+\frac{1}{C} x+f(x)=0
$$

where $R>0$ is the resistance, $L>0$ is the inductance, $C>0$ is the capacitance, and $f$ is a continuously differentiable function with respect to the charge $x$. Equation (2.4) can be transformed to the following equivalent system

$$
\left\{\begin{array}{l}
x_{1}^{\prime}=x_{2} \\
x_{2}^{\prime}=-\frac{1}{L C} x_{1}-\frac{R}{L} x_{2}-\frac{1}{L} f\left(x_{1}\right)
\end{array}\right.
$$

We now make the linear change of the variables as

$$
\left\{\begin{array}{l}
y=x_{1} \\
z=x_{2}+x_{1}
\end{array}\right.
$$

Then, by using (2.6), the system (2.5) can be written as

$$
\left\{\begin{array}{l}
y^{\prime}=z-y \\
z^{\prime}=\left(-\frac{1}{L C}+\frac{R}{L}-1\right) y+\left(-\frac{R}{L}+1\right) z-\frac{1}{L} f(y) .
\end{array}\right.
$$

Now we assume that the condition $0<C(R-L)<1$ holds. Then, it can be checked that

Moreover, we also assume that

$$
\frac{1}{L C}-\frac{R}{L}+1>0 \text { and } 1-\frac{R}{L}<0 .
$$

$$
f(0)=0, \text { and } 0<\frac{f(y)}{y}<2 A L, \quad \text { if } y \neq 0,
$$

where $A>0$ is a constant. Then we have

$$
0 \leqslant \frac{1}{L} \int_{0}^{y} f(\eta) d \eta=\frac{1}{L} \int_{0}^{y} \frac{f(\eta)}{\eta} \eta d \eta \leqslant \frac{2}{L} \int_{0}^{y}(A L) \eta d \eta=A y^{2} .
$$

Define a Lyapunov function by

$$
V(y, z)=\frac{1}{2}\left(\frac{1}{L C}-\frac{R}{L}+1\right) y^{2}+\frac{1}{2} z^{2}+\frac{1}{L} \int_{0}^{y} f(\eta) d \eta .
$$


It is clear that

$$
\frac{1}{3}\left(\frac{1}{L C}-\frac{R}{L}+1\right) y^{2}+\frac{1}{3} z^{2} \leqslant V(y, z) \leqslant\left(\frac{1}{L C}-\frac{R}{L}+A+1\right) y^{2}+z^{2} .
$$

If we put

$$
c_{1}=\min \left(\frac{1}{3}\left(\frac{1}{L C}-\frac{R}{L}+1\right), \frac{1}{3}\right), \quad c_{2}=\max \left(\left(\frac{1}{L C}-\frac{R}{L}+A+1\right), 1\right),
$$

then we have

$$
c_{1}\left\|(y, z)^{T}\right\|^{2} \leqslant V(t, y, z) \leqslant c_{2}\left\|(y, z)^{T}\right\|^{2} .
$$

For the time derivative $\dot{V}(y, z)$ of the Lyapunov function $V(y, z)$ along the solutions of system (2.7), we obtain

$$
\dot{V}(y, z)=-\left(\frac{1}{L C}-\frac{R}{L}+1\right) y^{2}+\left(1-\frac{R}{L}\right) z^{2}+\frac{1}{L}(-g(y) y) .
$$

Since $g(y) y \geqslant 0$ and $L>0$, we have

$$
\dot{V}(t, y, z) \leqslant-\left(\frac{1}{L C}-\frac{R}{L}+1\right) y^{2}-\left(\frac{R}{L}-1\right) z^{2} \leqslant-c_{3}\left(y^{2}+z^{2}\right)=-c_{3}\left\|(y, z)^{T}\right\|^{2},
$$

where

$$
c_{3}=\min \left(\left(\frac{1}{L C}-\frac{R}{L}+1\right),\left(\frac{R}{L}-1\right)\right) .
$$

Now, for the system (2.7) and the functions $g(t)=1+\sin (t)$ and $\alpha(t)=e^{-t}$, we consider the $P$-perturbed system

$$
\left\{\begin{array}{l}
y^{\prime}=\frac{1}{1+\sin (t)+e^{-t}}(z-y), \\
z^{\prime}=\frac{1}{1+\sin (t)+e^{-t}}\left(\left(-\frac{1}{L C}+\frac{R}{L}-1\right) y+\left(-\frac{R}{L}+1\right) z-\frac{1}{L} f(y)\right) .
\end{array}\right.
$$

If we assume that $c_{3} /\left(2 c_{2}\right)>1=\max (\cos (t))=g^{\prime}(t)$, then by using Theorem 3 we can conclude the solutions of the system (2.8) are uniform-ultimately Poisson bounded. Since (2.6) is a linear change of the variables, the solutions of the $P$ perturbed system

$$
\left\{\begin{array}{l}
x_{1}^{\prime}=\frac{1}{1+\sin (t)+e^{-t}} x_{2} \\
x_{2}^{\prime}=\frac{1}{1+\sin (t)+e^{-t}}\left(-\frac{1}{L C} x_{1}-\frac{R}{L} x_{2}-\frac{1}{L} f\left(x_{1}\right)\right)
\end{array}\right.
$$

with respect to the system (2.5) are uniform-ultimately Poisson bounded. 


\section{REFERENCES}

[1] A. P. Kartashev and B. L. Rozhdestvenskii, Ordinary differential equations and the foundations of the calculus of variations. Moscow: Nauka, 1980.

[2] K. S. Lapin, "Poisson total boundedness of solutions of systems of differential equations and Lyapunov vector functions," Mathematical Notes, vol. 104, no. 2, pp. 253-262, 2018, doi: 10.1134/S000143461807026X.

[3] K. S. Lapin, "Ultimate boundedness in the sense of Poisson of solutions to systems of Differential Equations and Lyapunov functions," Math. Notes, vol. 103, no. 2, pp. 221-231, 2018, doi: $10.1134 /$ S0001434618010236.

[4] K. S. Lapin, "Uniform boundedness in the sense of Poisson of solutions of systems of differential equations and Lyapunov vector functions," Differ. Equ., vol. 54, no. 1, pp. 38-48, 2018, doi: 10.1134/S0012266118010056.

[5] K. S. Lapin, "Vector Lyapunov functions and ultimate Poisson boundedness of solutions of systems of differential equations," Math. Notes, vol. 104, no. 1, pp. 63-73, 2018, doi: 10.1134/S0001434618070088.

[6] V. M. Matrosov, Method of Lyapunov vector functions: Analysis of dynamical properties of nonlinear systems. Moscow: Fizmatlit, 2001.

[7] T. Yoshizawa, "Liapunov's function and boundedness of solutions," Funkc. Ekvacioj. Ser. Int., no. 2, pp. 95-142, 1959.

Authors' addresses

Kirill S. Lapin

Mordovian State Pedagogical Institute named after M.E. Evseviev, Department of Informatics and Computer Engineering, Faculty of Mathematics and Physics, 11 A Studencheskaya St., 430010 Saransk, Russia.

E-mail address: klapin@mail.ru

\section{Cemil Tunç}

Van Yüzüncü Yil University, Department of Mathematics, Faculty of Sciences, 65080-Campus, Van-Turkey.

E-mail address: cemtunc@yahoo.com 